H. Fujimoto

Nagoya Math. J.

Vol. 170 (2003), 33-46

\title{
ON UNIQUENESS POLYNOMIALS FOR MEROMORPHIC FUNCTIONS
}

\author{
HIROTAKA FUJIMOTO
}

\begin{abstract}
A polynomial $P(w)$ is called a uniqueness polynomial (or a uiqueness polynomial in a broad sense) if $P(f)=c P(g)$ (or $P(f)=P(g)$ ) implies $f=g$ for any nonzero constant $c$ and nonconstant meromorphic functions $f$ and $g$ on C. We consider a monic polynomial $P(w)$ without multiple zero whose derivative has mutually distinct $k$ zeros $e_{j}$ with multiplicities $q_{j}$. Under the assumption that $P\left(e_{\ell}\right) \neq P\left(e_{m}\right)$ for all distinct $\ell$ and $m$, we prove that $P(w)$ is a uniqueness polynomial in a broad sense if and only if $\sum_{\ell<m} q_{\ell} q_{m}>\sum_{\ell} q_{\ell}$. We also give some sufficient conditions for uniqueness polynomials.
\end{abstract}

\section{$\S 1$. Introduction}

In this paper, a meromorphic function means a meromorphic function on the complex plane C. A discrete subset $S$ of $\mathbf{C}$ is called a uniqueness range set for meromorphic (or entire) functions if there exists no pair of two distinct nonconstant meromorphic (or entire) functions such that they have the same inverse images of $S$ counted with multiplicities. Since F. Gross and C. C. Yang proved that the set $S:=\left\{w ; w+e^{w}=0\right\}$ is a uniqueness range set for entire functions ([4]), many efforts were made to find uniqueness range sets which are as small as possible ([5], [9], [10]). In relation to this problem, B. Shiffman, C. C. Yang and X. Hua studied polynomials $P(w)$ satisfying the condition that there exists no pair of two distinct nonconstant meromorphic (or entire) functions $f$ and $g$ with $P(f)=P(g)$ in their papers [7] and [8]. For a finite set $S=\left\{a_{1}, a_{2}, \ldots, a_{q}\right\}$, it is necessary for $S$ to be a uniqueness range set for meromorphic (or entire) functions that the associated polynomial

$$
P_{S}(w)=\left(w-a_{1}\right)\left(w-a_{2}\right) \cdots\left(w-a_{q}\right)
$$

satisfies this condition.

In this paper, we use the following terminology.

Received May 21, 2001.

2000 Mathematics Subject Classification: 30D35. 
Definition 1.1. Let $P(w)$ be a nonconstant monic polynomial. We call $P(w)$ a uniqueness polynomial if $P(f)=c P(g)$ implies $f=g$ for any nonconstant meromorphic functions $f, g$ and any nonzero constant $c$. We also call $P(w)$ a uniqueness polynomial in a broad sense if $P(f)=P(g)$ implies $f=g$ for any nonconstant meromorphic functions $f, g$.

In the previous paper [1], the author gave some sufficient conditions for uniqueness polynomials as well as for uniqueness range sets.

Let $P(w)$ be a monic polynomial without multiple zero whose derivative has mutually distinct $k$ zeros $e_{1}, e_{2}, \ldots, e_{k}$ with multiplicities $q_{1}, q_{2}, \ldots, q_{k}$ respectively. Under the assumption that

$$
P\left(e_{\ell}\right) \neq P\left(e_{m}\right) \quad \text { for } 1 \leq \ell<m \leq k,
$$

he proved the following:

TheOREM 1.2. If $k \geq 4, P(w)$ is a uniqueness polynomial in a broad sense.

He also gave the following theorem for uniqueness polynomials:

TheOrem 1.3. For a polynomial $P(w)$ with $k \geq 4$ satisfying the hypothesis $(\mathrm{H})$, if

$$
P\left(e_{1}\right)+P\left(e_{2}\right)+\cdots+P\left(e_{k}\right) \neq 0
$$

then $P(w)$ is a uniqueness polynomial.

Moreover, he obtained some partial results for the case where $k=3$.

The main purpose of this paper is to give new geometric proofs of the above results in [1], which is due to the ideas used in [7, Section 4], and some improvements in [1] for the case where $k=2,3$.

We first investigate uniqueness polynomials in a broad sense. For a given nonconstant polynomial $P(z)$, we consider the algebraic curve $C$ in $P^{2}(\mathbf{C})$ which is the closur of a plane curve $\{(z, w) ;(P(z)-P(w)) /(z-w)=$ $0\}$ in $\mathbf{C}^{2}\left(\subset P^{2}(\mathbf{C})\right)$. We can show that $P(z)$ is a uniqueness polynomial in a broad sense if and only if every irreducible component of $C$ is of genus greater than one. Under the condition $(\mathrm{H})$, we prove that $C$ is irreducible and give a formula for the genus of $C$. These enable us to obtain the following improvement of the above results: 
THEOREM 1.4. Let $P(w)$ be a polynomial satisfying the above assumption $(\mathrm{H})$. Then, $P(w)$ is a uniqueness polynomial in a broad sense if and only if

$$
\sum_{1 \leq \ell<m \leq k} q_{\ell} q_{m}>\sum_{\ell=1}^{k} q_{\ell} .
$$

We can show that, for the case $k \geq 4$, the condition (1.5) is always satisfied. Moreover, (1.5) holds when $\max \left(q_{1}, q_{2}, q_{3}\right) \geq 2$ for the case $k=3$ and when $\min \left(q_{1}, q_{2}\right) \geq 2$ and $q_{1}+q_{2} \geq 5$ for the case $k=2$.

Next, we try to obtain some improvements of the results in [1] for uniqueness polynomials with $k=3$. We prove the following:

TheOREM 1.6. Let $P(w)$ be a monic polynomial with $k=3$ satisfying the condition $(\mathrm{H})$. Assume that $\max \left(q_{1}, q_{2}, q_{3}\right) \geq 2$ and

$$
\begin{gathered}
\frac{P\left(e_{\ell}\right)}{P\left(e_{m}\right)} \neq \pm 1 \quad \text { for } 1 \leq \ell<m \leq 3, \\
\frac{P\left(e_{\ell}\right)}{P\left(e_{m}\right)} \neq \frac{P\left(e_{m}\right)}{P\left(e_{n}\right)} \quad \text { for any permutation }(\ell, m, n) \text { of }(1,2,3) .
\end{gathered}
$$

Then, $P(w)$ is a uniqueness polynomial.

Lastly, we give some sufficient conditions for uniqueness polynomial for the case $k=2$, which is not treated in [1].

\section{$\S 2$. Uniqueness polynomials in a broad sense}

Let $P(w)$ be a monic polynomial of degree $q(>0)$ without multiple zero, and let its derivative be given by

$$
P^{\prime}(w)=q\left(w-e_{1}\right)^{q_{1}}\left(w-e_{2}\right)^{q_{2}} \ldots\left(w-e_{k}\right)^{q_{k}},
$$

where $e_{1}, \ldots, e_{k}$ are mutually distinct and $q_{1}+q_{2}+\cdots+q_{k}=q-1$.

In the followings, we assume $k \geq 2$, because $P(w)$ cannot be a uniqueness polynomial in a broad sense for the case $k=1$ (cf., [1, p. 1183]). Furthermore, by technical reasons we assume the following:

$$
P\left(e_{\ell}\right) \neq P\left(e_{m}\right) \quad \text { for } 1 \leq \ell<m \leq k .
$$


Consider the polynomial

$$
Q(z, w):=\frac{P(z)-P(w)}{z-w}
$$

in two variables $z$ and $w$, and the associated homogeneous polynomial

$$
Q^{*}\left(u_{0}, u_{1}, u_{2}\right):=u_{0}^{d} Q\left(\frac{u_{1}}{u_{0}}, \frac{u_{2}}{u_{0}}\right)
$$

of degree $d$ in three variables $u_{0}, u_{1}, u_{2}$, where $d:=q-1$. By using this, we define the algebraic curve

$$
C: Q^{*}\left(u_{0}, u_{1}, u_{2}\right)=0, \quad\left(u_{0}: u_{1}: u_{2}\right) \in P^{2}(\mathbf{C}),
$$

where $\left(u_{0}: u_{1}: u_{2}\right)$ denote the homogeneous coordinates on $P^{2}(\mathbf{C})$.

Proposition 2.3. The algebraic curve $C$ has ordinary singularities with multiplicities $q_{\ell}$ at the points $P_{\ell}:=\left(1: e_{\ell}: e_{\ell}\right)(1 \leq \ell \leq k)$, and has regular points at all other points.

Proof. Set $L_{\infty}:=\left\{u_{0}=0\right\}$. We first investigate points in $C \cap L_{\infty}$. By the assumption, $P(w)$ can be written as

$$
P(w)=w^{d+1}+\text { terms of lower degree }
$$

and so we have

$$
Q^{*}\left(u_{0}, u_{1}, u_{2}\right)=\left(u_{1}^{d}+u_{1}^{d-1} u_{2}+\cdots+u_{2}^{d}\right)+u_{0} R\left(u_{0}, u_{1}, u_{2}\right),
$$

where $R\left(u_{0}, u_{1}, u_{2}\right)$ is a homogeneous polynomial of degree $d-1$. It is easily seen that the first term is factorized into mutually distinct $d$ linear functions $u_{1}-\zeta^{\ell} u_{2}(\ell=1,2, \ldots, d)$, where $\zeta$ denotes a primitive $(d+1)$-st root of unity. This shows that $C \cap L_{\infty}$ consists of mutually distinct $d$ points $Q_{\ell}:=\left(0: \zeta^{\ell}: 1\right)(\ell=1,2, \ldots, d)$ and each $Q_{\ell}$ is a regular point of $C$.

We next investigate the singularities of $C \backslash L_{\infty}$. We may use inhomogeneous coodinates $z, w$. Let $P_{0}=\left(z_{0}, w_{0}\right)\left(=\left(1: z_{0}: w_{0}\right)\right)$ be a singularity of $C$, namely, let $P_{0}$ satisfy the condition

$$
Q\left(z_{0}, w_{0}\right)=Q_{z}\left(z_{0}, w_{0}\right)=Q_{w}\left(z_{0}, w_{0}\right)=0 .
$$

Then, by differentiating the identity

$$
P(z)-P(w)=(z-w) Q(z, w),
$$


we have

$q\left(z_{0}-e_{1}\right)^{q_{1}}\left(z_{0}-e_{2}\right)^{q_{2}} \cdots\left(z_{0}-e_{k}\right)^{q_{k}}=\left(z_{0}-w_{0}\right) Q_{z}\left(z_{0}, w_{0}\right)+Q\left(z_{0}, w_{0}\right)=0$.

This implies that $z_{0}=e_{\ell}$ for some $\ell(1 \leq \ell \leq k)$. By the same reason, we see $w_{0}=e_{m}$ for some $m$. It then follows that

$$
P\left(e_{\ell}\right)-P\left(e_{m}\right)=\left(z_{0}-w_{0}\right) Q\left(z_{0}, w_{0}\right)=0
$$

By virtue of the assumption $(\mathrm{H})$, we can conclude $\ell=m$. Therefore, $C$ has no singulalrities outside $P_{\ell}$ 's.

We next investigate shapes of $C$ around each point $P_{\ell}$. Without loss of generality, we may assume $\ell=1$ and $e_{1}=0$ after suitable translations of coordinates. Then, by the assumption (2.1), we can write

$$
P(w)-P\left(e_{1}\right)=c w^{q_{1}+1}+\text { terms of higher degree }
$$

with a nonzero constant $c$, and so

$$
Q(z, w)=c\left(z^{q_{1}}+z^{q_{1}-1} w+\cdots+w^{q_{1}}\right)+\text { terms of higher degree. }
$$

The first term in this expansion can be factorized into the product of mutually distinct linear forms $z-\eta^{\ell} w\left(\ell=1,2, \ldots, q_{1}\right)$ in $z$ and $w$, where $\eta$ denotes a primitive $\left(q_{1}+1\right)$-st root of unity. This shows that $P_{1}$ is an ordinary singularity of $C$ with multiplicity $q_{1}$ (cf., [2, p. 66]). The proof of Proposition 2.3 is completed.

Proposition 2.4. The curve $C$ is irreducible.

Proof. Suppose that $C$ is reducible and so $Q(z, w)$ can be written as

$$
Q(z, w)=Q_{1}(z, w) Q_{2}(z, w)
$$

with nonconstant polynomials $Q_{1}$ and $Q_{2}$. Consider the curves

$$
C_{i}: Q_{i}^{*}\left(u_{0}, u_{1}, u_{2}\right):=u_{0}^{d_{i}} Q_{i}\left(\frac{u_{1}}{u_{0}}, \frac{u_{2}}{u_{0}}\right)=0, \quad(i=1,2)
$$

in $P^{2}(\mathbf{C})$, where each $d_{i}$ denotes the degree of $C_{i}$. We then have

$$
C_{1} \cap C_{2} \subseteq\left\{P_{1}, P_{2}, \ldots, P_{k}\right\},
$$


because $C$ has a singularity at every point in $C_{1} \cap C_{2}$. Since $C_{1} \cap C_{2}$ is discrete, $C_{1}$ and $C_{2}$ have no common irreducible component. For each $\ell$, there is a neighborhood $U$ of $P_{\ell}$ such that $U \cap C$ has mutually distinct $q_{\ell}$ irreducible components by virtue of Hensel's lemma (cf., [6, p. 68]). Some of them are included in $C_{1}$ and the others are included in $C_{2}$. These guarantee that $C_{i}$ has at worst ordinary singularities at some of the points $P_{\ell}$ 's and regular points elsewhere. Assume that $C_{1}$ and $C_{2}$ have ordinary singularities of multiplicities $r_{\ell}$ and $s_{\ell}\left(0 \leq r_{\ell}, s_{\ell} \leq q_{\ell}\right)$ at each $P_{\ell}$ respectively, where an ordinary singularity of multiplicity 0 means that the curve does not contain $P_{\ell}$. We then have

$$
q_{\ell}=r_{\ell}+s_{\ell} \quad(\ell=1,2, \ldots, k) .
$$

Moreover, we can show

$$
d_{1}=r_{1}+r_{2}+\cdots+r_{k}, \quad d_{2}=s_{1}+s_{2}+\cdots+s_{k}
$$

To see this, we consider the diagonal line

$$
L_{\Delta}: u_{1}-u_{2}=0
$$

in $P^{2}(\mathbf{C})$. Since

$$
Q(z, z)=\lim _{w \rightarrow z} Q(z, w)=\lim _{w \rightarrow z} \frac{P(w)-P(z)}{w-z}=P^{\prime}(z)
$$

we have $C_{1} \cap L_{\Delta} \subseteq\left\{P_{1}, P_{2}, \ldots, P_{k}\right\}$. The tangent lines

$$
z-e_{\ell}-\eta^{\ell}\left(w-e_{\ell}\right)=0
$$

of $C$ at $P_{\ell}$ do not coincide with $L_{\Delta}$, and so the intersection number of $C_{1}$ and $L_{\Delta}$ at $P_{\ell}$ is $r_{\ell}$. By the classical Bezout's theorem (cf., [2, p. 112]), we get

$$
d_{1}=r_{1}+r_{2}+\cdots+r_{k} .
$$

Similarly, we have $d_{2}=s_{1}+s_{2}+\cdots+s_{k}$.

On the other hand, the intersection number of $C_{1}$ and $C_{2}$ at each point $P_{\ell}$ is $r_{\ell} s_{\ell}$. Applying Bezout's theorem again, we obtain

$$
d_{1} d_{2}=r_{1} s_{1}+r_{2} s_{2}+\cdots+r_{k} s_{k}
$$

Therefore,

$$
\sum_{\ell, m} r_{\ell} s_{m}-\sum_{\ell} r_{\ell} s_{\ell}=\sum_{\ell \neq m} r_{\ell} s_{m}=0
$$


Since $r_{\ell}$ and $s_{\ell}$ are nonnegative integers, we have necessarily $r_{\ell} s_{m}=0$ for all mutually distinct $\ell$ and $m$. Changing indices if necessary, we may assume $r_{1} \neq 0$, because $d_{1}=\sum r_{\ell}>0$. Then, $s_{\ell}=0$ for $\ell=2,3, \ldots, k$. On the other hand, since $d_{2}=\sum s_{\ell}>0$, we see $s_{1} \neq 0$. This implies that $r_{\ell}=0$ for $\ell=2, \ldots, k$, because $s_{1} r_{\ell}=0$ for $\ell=2, \ldots, k$. By (2.5), this shows that $k=1$, which contradicts the assumption $k \geq 2$. Proposition 2.4 is completely proved.

With each irreducible algebraic curve $V$ in $P^{2}(\mathbf{C})$ we can associate the normalization $(\widetilde{V}, \mu)$ of $V$, namely, a compact Riemann surface $\widetilde{V}$ and a holomorphic mapping $\mu$ of $\widetilde{V}$ onto $V$ which is injective outside the inverse image of the singular locus of $V$. By definition, the genus $g(V)$ of $V$ means the genus of the compact Riemann surface $\widetilde{V}$.

Proposition 2.7. The genus of the curve $C$ defined as above is given by

$$
g(C)=\frac{(d-1)(d-2)}{2}-\sum_{\ell=1}^{k} \frac{q_{\ell}\left(q_{\ell}-1\right)}{2} .
$$

This is an easy result of Propositions 2.3, 2.4 and the classical Plücker's genus formula (cf., [2, p. 199]).

THEOREM 2.8. Let $P(w)$ be a monic polynomial whose derivative has $k$ distinct zeros $e_{1}, e_{2}, \ldots, e_{k}$ with multiplicities $q_{1}, q_{2}, \ldots, q_{k}$, respectivley. Assume that

$$
P\left(e_{\ell}\right) \neq P\left(e_{m}\right), \quad(1 \leq \ell<m \leq k) .
$$

If $k \geq 4$, then $P(w)$ is a uniqueness polynomial in a broad sense.

Moreover, $P(w)$ is a uniqueness polynomial in a broad sense when and only when

$$
\max \left(q_{1}, q_{2}, q_{3}\right) \geq 2
$$

for the case $k=3$, and when and only when

$$
\min \left(q_{1}, q_{2}\right) \geq 2 \quad \text { and } \quad q_{1}+q_{2} \geq 5
$$

for the case $k=2$.

Remark. (1) In [1], the author proved Theorem 2.8 for the case $k \geq 4$ and the 'when' part for the case $k=3$ under the additional assumption $\left(e_{1}, e_{2}, e_{3}, \infty\right)=-1$ by function-theoretic method. 
(2) For the case $k=2$, there is no harm in assuming that $e_{1}=0$ and $e_{2}=1$ after a suitable linear change of coordinate on $\mathbf{C}$. In this case, $P(w)$ is nothing but the polynomial studied by Frank and Reinders in [3] after a suitable multiplication of a nonzero constant. In this particular case, the condition $(\mathrm{H})$ is automatically satisfied, because

$$
(-1)^{q_{2}}(P(1)-P(0))=\int_{0}^{1} q x^{q_{1}}(1-x)^{q_{2}} d x>0 .
$$

In [3], Frank and Reinders proved Theorem 2.8 for a particular case where $k=2, \min \left(q_{1}, q_{2}\right)=2$ and $q_{1}+q_{2} \geq 6$.

Proof. Suppose that $P(w)$ is not a uniqueness polynomial in a broad sense. By definition, there exist two distinct nonconstant meromorphic functions $f$ and $g$ satisfying the condition $P(f)=P(g)$. We can write $f=f_{1} / f_{0}$ and $g=f_{2} / f_{0}$ with suitably chosen entire functions $f_{0}, f_{1}, f_{2}$ without common zeros. Consider a holomorphic map

$$
\Phi:=\left(f_{0}: f_{1}: f_{2}\right): \mathbf{C} \longrightarrow P^{2}(\mathbf{C}) .
$$

We denote by $E$ the union of the sets of all poles of $f$, of all poles of $g$ and of all points $z$ with $f(z)=g(z)$. By the assumption, $E$ is a discrete subset of $\mathbf{C}$, and we have

$$
\Phi(\mathbf{C} \backslash E) \subseteq\left\{(z, w) \in P^{2}(\mathbf{C}) \backslash L_{\infty} ; Q(z, w):=\frac{P(z)-P(w)}{z-w}=0\right\} .
$$

Therefore, by the continuity of $\Phi$ the image $\Phi(\mathbf{C})$ is included in the algebraic curve $C$ defined by $(2.2)$. Take the normalization $(\widetilde{C}, \mu)$ of $C$. Then, there is a nonconstant holomorphic map $\widetilde{\Phi}$ of $\mathbf{C}$ into $\widetilde{C}$ with $\mu \cdot \widetilde{\Phi}=\Phi$. For our purpose, it suffices to seek the condition for the genus $g(\widetilde{C})(=g(C))$ of the compact Riemann surface $\widetilde{C}$ is greater than one. In fact, in this case, we have an absurd conclusion that the map $\widetilde{\Phi}$, and so $\Phi$, is a constant by virtue of the classical Picard's theorem, which asserts that every holomorphic map of $\mathbf{C}$ into a compact Riemann surface of genus greater than one is necessarily a constant. On the other hand, if $g(\widetilde{C})$ is not larger than one, then $\widetilde{C}$ is a torus or the Riemann sphere. Therefore, there exists a nonconstant holomorphic map $\widetilde{\Psi}$ of $\mathbf{C}$ into $\widetilde{C}$. Consider the map $\Psi:=\mu \cdot \widetilde{\Psi}$, which can be regarded as a holomorphic map of $\mathbf{C}$ into $P^{2}(\mathbf{C})$. We write $\Psi=$ $\left(f_{0}^{*}: f_{1}^{*}: f_{2}^{*}\right)$ with nonzero holomorphic functions which have no common zeros. It is easily seen that $f^{*}:=f_{1}^{*} / f_{0}^{*}$ and $g^{*}:=f_{2}^{*} / f_{0}^{*}$ are nonconstant 
distinct meromorphic functions satisfying the condition $P\left(f^{*}\right)=P\left(g^{*}\right)$. The polynomial $P(w)$ cannot be a uniqueness polynomial in a broad sense. On the other hand, according to Proposition 2.7 the genus of $C$ is given by

$$
g(C)=\frac{(d-1)(d-2)}{2}-\sum_{\ell=1}^{k} \frac{q_{\ell}\left(q_{\ell}-1\right)}{2}=\sum_{1 \leq \ell<m \leq k} q_{\ell} q_{m}-\sum_{\ell=1}^{k} q_{\ell}+1(\geq 0) .
$$

Therefore, $P(w)$ is a uniqueness polynomial in a broad sense if and only if it satisfies the condition (1.5) as mentioned in Section 1.

For the case $k \geq 4$, it is easily seen that

$$
g(C)=q_{1}\left(\sum_{\ell=2}^{k} q_{\ell}-1\right)+\left(\sum_{2 \leq \ell<m \leq k} q_{\ell} q_{m}-\sum_{\ell=2}^{k} q_{\ell}+1\right) \geq k-2 \geq 2 .
$$

For the case $k=3$, under the assumption that at least one of $q_{\ell}$ 's is larger than one, say $q_{3} \geq 2$, we have

$$
g(C)=q_{1}\left(q_{2}+q_{3}-1\right)+\left(q_{2}-1\right)\left(q_{3}-1\right) \geq 2 .
$$

Moreover, for the case $k=2$, under the assumption $\min \left(q_{1}, q_{2}\right) \geq 2$ and $q_{1}+q_{2} \geq 5$, we have

$$
g(C)=\left(q_{1}-1\right)\left(q_{2}-1\right) \geq 2 .
$$

Conversely, for the case $k=3$, if $q_{1}=q_{2}=q_{3}=1$, we have $g(C)=1$. For the case $k=2, q_{1}=1, q_{2}=1$ or $q_{1}+q_{2} \leq 4$, then $g(C) \leq 1$. The proof of Theorem 2.8 is completed.

\section{§3. Uniqueness polynomials}

As in the previous section, we consider a monic polynomial $P(w)$ without multiple zero whose derivative has mutually distinct $k(>1)$ zeros $e_{1}, e_{2}, \ldots, e_{k}$ with multiplicities $q_{1}, q_{2}, \ldots, q_{k}$ respectively, and assume that $P(w)$ satisfies the condition $(\mathrm{H})$.

In the previous paper [1], the author proved the following:

Theorem 3.1. Assume that $k \geq 4$. If $P(w)$ is not a uniqueness polynomial, then there is a permutation $\left(i_{1}, i_{2}, \ldots, i_{k}\right)$ of $(1,2, \ldots, k)$ such that

$$
\frac{P\left(e_{i_{1}}\right)}{P\left(e_{1}\right)}=\frac{P\left(e_{i_{2}}\right)}{P\left(e_{2}\right)}=\cdots=\frac{P\left(e_{i_{k}}\right)}{P\left(e_{k}\right)} \neq 1 \text {. }
$$


We note that Theorem 1.3 mentioned in Section 1 is an immediate consequence of Theorem 3.1.

We now investigate the polynomial with $k=3$. Changing indices if necessary, we assume that $q_{1} \leq q_{2} \leq q_{3}$.

THEOREM 3.2. Assume that $P(w)$ with $k=3$ is not a uniqueness polynomial.

(1) If $q_{1} \geq 2$, then $P(w)$ satisfies the condition

$$
\frac{P\left(e_{i_{1}}\right)}{P\left(e_{1}\right)}=\frac{P\left(e_{i_{2}}\right)}{P\left(e_{2}\right)}=\frac{P\left(e_{i_{3}}\right)}{P\left(e_{3}\right)} \neq 1 .
$$

for some permutation $\left(i_{1}, i_{2}, i_{3}\right)$ of the indices $(1,2,3)$.

(2) If $q_{1}=1$ and $2 \leq q_{2} \leq q_{3}$, then $P(w)$ satisfies the condition (C1) or

$$
P\left(e_{2}\right)+P\left(e_{3}\right)=0
$$

(3) If $q_{1}=q_{2}=1$ and $q_{3} \geq 2$, then $P(w)$ satisfies the condition (C1) or

$$
P\left(e_{1}\right)+P\left(e_{3}\right)=0, \quad P\left(e_{2}\right)+P\left(e_{3}\right)=0 \text { or } P\left(e_{1}\right) P\left(e_{2}\right)=P\left(e_{3}\right)^{2}
$$

For the proof of Theorem 3.2, we assume that there are distinct nonconstant meromorphic functions $f$ and $g$ and a nonzero constant $c$ such that $P(f)=c P(g)$. For all cases of Theorem 3.2, the assumptions of Theorem 2.8 are satisfied and so $P(w)$ is a uniqueness polynomial in a broad sense. Therefore, we have necessarily $c \neq 1$. As in the previous paper ([1]), we set

$$
\Lambda:=\left\{(\ell, m) ; P\left(e_{\ell}\right)=c P\left(e_{m}\right)\right\} .
$$

We give the following lemma, which is an improvement of [1, Lemma 5.3].

Lemma 3.3. Assume that $k=3$ and $q_{\ell_{0}} \geq 2$ for some $\ell_{0}$. Then, there are some indices $m$ and $m^{\prime}$ such that $\left(\ell_{0}, m\right) \in \Lambda$ and $\left(m^{\prime}, \ell_{0}\right) \in \Lambda$.

Proof. This is proved by the same argument as in the proof of Lemma 5.3 of [1] with some simple modifications. For reader's convenience, we state the outline of the proof. We assume that $\left(\ell_{0}, m\right) \notin \Lambda$ for any $m$. For each point $z_{0}$ with $f\left(z_{0}\right)=e_{\ell_{0}}$, we see $g\left(z_{0}\right) \neq e_{m}$ for any $m$. Since $P^{\prime}(f) f^{\prime}=c P^{\prime}(g) g^{\prime}$, we have necessarily $g^{\prime}\left(z_{0}\right)=0$. This implies that 
$N\left(r, \nu_{f}^{e_{\ell_{0}}}\right) \leq N\left(r,\left.\nu_{g^{\prime}}^{*}\right|_{f=e_{\ell_{0}}}\right)$. Here, $N\left(r, \nu_{f}^{e_{\ell_{0}}}\right)$ and $N\left(r,\left.\nu_{g^{\prime}}^{*}\right|_{f=e_{\ell_{0}}}\right)$ denote the counting functions of zeros of $f-e_{\ell_{0}}$ counted with multiplicities and of zeros $z$ of $g^{\prime}$ counted with multiplicities such that $f(z)=e_{\ell_{0}}$ and $g(z) \neq e_{m}$ for any $m$, respectively. Assume that there are constants $c_{0}(\neq 0)$ and $c_{1}$ with $g=c_{0} f+c_{1}$. Then, the assumption $P(f)=c P(g)$ implies

$$
\begin{aligned}
& \left(f-e_{1}\right)^{q_{1}}\left(f-e_{2}\right)^{q_{2}}\left(f-e_{3}\right)^{q_{3}} \\
& \quad=c c_{0}\left(c_{0} f+c_{1}-e_{1}\right)^{q_{1}}\left(c_{0} f+c_{1}-e_{2}\right)^{q_{2}}\left(c_{0} f+c_{1}-e_{3}\right)^{q_{3}} .
\end{aligned}
$$

Since $f$ is not a constant, this is regarded as an identity of polynomials with indeterminate $f$. Using the unique factorization theorem as in [1, p. 1191], we can easily show that, for every $\ell$, there is some $m$ with $(\ell, m) \in \Lambda$, which contradicts the assumption. Hence, there does not exist such constants $c_{0}$ and $c_{1}$. As in $\left[1\right.$, p. 1184], we set $k_{0}=\# \Lambda$. By the assumption, we see $k_{0} \leq 2$, and so we can apply Lemma 3.8 of [1] to obtain $N\left(r,\left.\nu_{g^{\prime}}^{*}\right|_{f=e_{\ell_{0}}}\right)=$ $S(r, f)+S(r, g)$. Therefore, $N\left(r, \nu_{f}^{e_{\ell}}\right)=S(r, f)+S(r, g)$.

Consider the polynomial $Q(w):=P(w)-P\left(e_{\ell_{0}}\right)$ and $Q^{*}(w):=c P(w)-$ $P\left(e_{\ell_{0}}\right)$. We denote all distinct zeros of $Q(w)$ and $Q^{*}(w)$ by $\alpha_{1}\left(=e_{\ell_{0}}\right)$, $\alpha_{2}, \ldots, \alpha_{m}$ and $\beta_{1}, \beta_{2}, \ldots, \beta_{n}$, respectively. Since $Q$ has a zero of multiplicity $q_{\ell_{0}}+1$ at $\alpha_{1}$, we have $m \leq q-q_{\ell_{0}} \leq q-2$. Moreover, each $\beta_{j}$ $(1 \leq j \leq n)$ is not equal to $e_{m}$ for any $m$, because $Q^{*}\left(e_{m}\right)=0$ implies $\left(\ell_{0}, m\right) \in \Lambda$. This shows that all $\beta_{j}$ 's are simple zeros of $Q^{*}(w)$ and so $n=q$. On the other hand, if $g=\beta_{j}$ for some $j$ at a point $z_{0}$, then $P\left(f\left(z_{0}\right)\right)=c P\left(g\left(z_{0}\right)\right)=c P\left(\beta_{j}\right)=P\left(e_{\ell_{0}}\right)$ and so $f\left(z_{0}\right)=\alpha_{i}$ for some $i$. By the second main theorem in value distribution theory, we obtain

$$
\begin{aligned}
(q-2) T(r, g) & \leq \sum_{j=1}^{q} N\left(r, \bar{\nu}_{g}^{\beta_{j}}\right)+S(r, g) \\
& \leq N\left(r, \bar{\nu}_{f}^{e_{\ell_{0}}}\right)+\sum_{i=2}^{m} N\left(r, \bar{\nu}_{f}^{\alpha_{i}}\right)+S(r, g) \\
& \leq(m-1) T(r, f)+S(r, g),
\end{aligned}
$$

where $N\left(r, \bar{\nu}_{g}^{\beta_{j}}\right)$ denotes the counting functions of the points $z$ with $g(z)=$ $\beta_{j}$ counted without multiplicities. This gives an absurd conclusion $q-2 \leq$ $m-1 \leq q-3$. Therefore, there is some $m$ with $\left(\ell_{0}, m\right) \in \Lambda$. The proof of the existence of $m^{\prime}$ with $\left(m^{\prime}, \ell_{0}\right)$ is similar. Thus, we get Lemma 3.3.

Now, we start to inquire into the assertion (1) of Theorem 3.2, namely, the case $\min \left(q_{1}, q_{2}, q_{3}\right) \geq 2$. By Lemma 3.3 there are indices $i_{1}, i_{2}, i_{3}$ such 
that $\left(\ell, i_{\ell}\right) \in \Lambda(\ell=1,2,3)$. In this situation, it is easily seen that these $i_{1}, i_{2}, i_{3}$ are mutually distinct by Lemma 3.5 of [1]. As its consequence, we have the desired conclusion for the case (1).

We next inquire into the assertion (2), namely, the case $q_{1}=1$ and $2 \leq q_{2} \leq q_{3}$. In this case, there are indices $i_{2}, i_{3}$ and $j_{2}, j_{3}$ such that

$$
\left(2, i_{2}\right) \in \Lambda, \quad\left(3, i_{3}\right) \in \Lambda, \quad\left(j_{2}, 2\right) \in \Lambda, \quad\left(j_{3}, 3\right) \in \Lambda .
$$

If $\min \left(i_{2}, i_{3}\right) \geq 2$, then we have necessarily $i_{2}=3$ and $i_{3}=2$ by Lemma 3.5 of [1] because $c \neq 1$. Therefore, we get

$$
c=\frac{P\left(e_{2}\right)}{P\left(e_{3}\right)}=\frac{P\left(e_{3}\right)}{P\left(e_{2}\right)},
$$

and so $P\left(e_{2}\right)^{2}=P\left(e_{3}\right)^{2}$. Since $P\left(e_{2}\right) \neq P\left(e_{3}\right)$ by the assumption $(\mathrm{H})$, we have the conclusion (C2). It remains to consider the case $i_{2}=1$ or $i_{3}=1$. Changing indices if necessary, we assume that $i_{2}=1$, namely, $(2,1) \in \Lambda$. This implies that $i_{3}=2$, namely, $(3,2) \in \Lambda$, because $i_{3} \neq 1,3$ by Lemma 3.5 of $[1]$ and the fact $c \neq 1$. Moreover, we have $(1,3) \in \Lambda$ by the same reason. Therefore, we have (C1).

Lastly, we inquire into the assertion (3), namely, the case $q_{1}=q_{2}=1$ and $q_{3} \geq 2$. In this case, there are indices $i$ and $j$ such that $(3, i) \in \Lambda$ and $(j, 3) \in \Lambda$. Then, we may assume $i=1$ and so $(3,1) \in \Lambda$ by exchanging the role of indices 1 and 2 if necessary. If $j=1$, then we have $P\left(e_{1}\right)+P\left(e_{3}\right)=0$ and, if $j=2$, then we have $P\left(e_{1}\right) P\left(e_{2}\right)=P\left(e_{3}\right)^{2}$. The proof of Theorem 3.2 is completed.

We note here that Theorem 1.6 mentioned in Section 1 is an easy consequence of Theorem 3.2.

For the case $k=2$, we can prove the following:

THEOREM 3.4. Assume that the derivative $P^{\prime}(w)$ has two distinct zeros $e_{1}$ and $e_{2}$ with multiplicities $q_{1}$ and $q_{2}$ respectively and assume that $q_{1} \leq q_{2}$. If it satisfies one of the conditions

(1) $q_{1} \geq 3$ and $P\left(e_{1}\right)+P\left(e_{2}\right) \neq 0$,

(2) $q_{1} \geq 2$ and $q_{2} \geq q_{1}+3$,

then $P(w)$ is a uniqueness polynomial.

Proof. Assume that $P(w)$ is not a uniqueness polynomial. Then, there are nonconstant distinct meromorphic functions $f, g$ and a nonzero constant $c$ such that $P(f)=c P(g)$. By virtue of Theorem 2.8 we have $c \neq 1$.

We first show the following: 
Lemma 3.5. If $c \neq P\left(e_{2}\right) / P\left(e_{1}\right)$, then $q_{2} \leq 2$.

Proof. As in the proof of Lemma 3.3, we consider the polynomials $Q(w):=P(w)-P\left(e_{2}\right)$ and $Q^{*}(w):=c P(w)-P\left(e_{2}\right)$, and denote all zeros of $Q(w)$ and $Q^{*}(w)$ by $\alpha_{1}\left(=e_{2}\right), \alpha_{2}, \ldots, \alpha_{m}$ and $\beta_{1}, \beta_{2}, \ldots, \beta_{n}$, respectively. Then, $\alpha_{1}$ is a zero of $Q(w)$ with multiplicity $q_{2}+1$ and $\alpha_{i}$ are simple zeros of it for $i=2,3, \ldots, m$. Moreover, by the assumption, all $\beta_{j}(1 \leq j \leq n)$ are simple zeros of $Q^{*}(w)$. Therefore, $m=q-q_{2}=q_{1}+1$ and $n=q$. We now apply the second main theorem to the function $g$ and $q$ values $\beta_{j}$ 's to obtain

$$
(q-2) T(r, g) \leq \sum_{j=1}^{q} N\left(r, \bar{\nu}_{g}^{\beta_{j}}\right)+S(r, g),
$$

For every point $z_{0}$ with $g\left(z_{0}\right)=\beta_{j}$, we have $P\left(f\left(z_{0}\right)\right)=c P\left(g\left(z_{0}\right)\right)=$ $c P\left(\beta_{j}\right)=P\left(e_{2}\right)$ and so $f\left(z_{0}\right)$ is equal to one of the values $\alpha_{1}, \alpha_{2}, \ldots, \alpha_{m}$. Noting that $T(r, f)=T(r, g)+O(1)$ by Lemma 3.2 of [1], we obtain

$$
\begin{aligned}
(q-2) T(r, g) & \leq \sum_{j=1}^{m} N\left(r, \bar{\nu}_{f}^{\alpha_{j}}\right)+S(r, f) \\
& \leq m T(r, f)+S(r, f) \\
& \leq\left(q_{1}+1\right) T(r, g)+S(r, g) .
\end{aligned}
$$

This concludes that $q-2=q_{1}+q_{2}+1-2 \leq q_{1}+1$, whence $q_{2} \leq 2$.

We continue the proof of Theorem 3.4. Under the assumption of (1), we have either $c \neq P\left(e_{2}\right) / P\left(e_{1}\right)$ or $c \neq P\left(e_{1}\right) / P\left(e_{2}\right)$, because otherwise

$$
c^{2}=\frac{P\left(e_{2}\right)}{P\left(e_{1}\right)} \frac{P\left(e_{1}\right)}{P\left(e_{2}\right)}=1,
$$

which contradicts to the assumption $P\left(e_{1}\right)+P\left(e_{2}\right) \neq 0$. Therefore, $q_{1} \leq 2$ or $q_{2} \leq 2$ as a consequence of Lemma 3.5. Thus, we have the assertion (1).

The proof of the assertion (2) is given by the the same argument as in [3, 191]. For readers' convenience, we repeat it here. By virtue of Lemma 3.5, it suffices to consider the only case $c=P\left(e_{2}\right) / P\left(e_{1}\right)$. By the same argument as in the proof of Lemma 3.5, $Q(w):=P(w)-P\left(e_{2}\right)$ has mutually distinct $m:=q_{1}+1$ zeros $\alpha_{1}, \ldots, \alpha_{m}$ and $Q^{*}(w):=c P(w)-P\left(e_{2}\right)$ has mutually distinct $n:=q_{2}+1$ zeros $\beta_{1}, \ldots, \beta_{n}$. In this case, if $g\left(z_{0}\right)=\beta_{j}$ for some 
$z_{0} \in \mathbf{C}$ and some $j$, then $f\left(z_{0}\right)=\alpha_{i}$ for some $i$. Therefore, we have

$$
\begin{aligned}
\left(\left(q_{2}+1\right)-2\right) T(r, g) & \leq \sum_{j=1}^{m} N\left(r, \bar{\nu}_{g}^{\beta_{j}}\right)+S(r, g) \\
& \leq \sum_{i=1}^{m} N\left(r, \bar{\nu}_{f}^{\alpha_{i}}\right)+S(r, g) \\
& \leq\left(q_{1}+1\right) T(r, g)+S(r, g) .
\end{aligned}
$$

This concludes $q_{2}-1 \leq q_{1}+1$, which contradicts the assumption. The proof of Theorem 3.4 is completed.

\section{REFERENCES}

[1] H. Fujimoto, On uniqueness of meromorphic functions sharing finite sets, Amer. J. Math., 122 (2000), 1175-1203.

[2] W. Fulton, Algebraic curves, W. A. Benjamin, New York, 1969.

[3] G. Frank and M. Reinders, A unique range set for meromorphic functions with 11 elements, Complex Variables, 37 (1998), 185-193.

[4] F. Gross and C. C. Yang, On preimage and range sets of meromorphic functions, Proc. Japan Acad., 58 (1982), 17-20.

[5] P. Li and C. C. Yang, Some further results on the unique range sets of meromorphic functions, Kodai Math. J., 18 (1995), 437-450.

[6] R. Miranda, Algebraic curves and Riemann surfaces, Amer. Math. Soc., 1995.

[7] B. Shiffman, Uniqueness of entire and meromorphic functions sharing finite sets, Complex Variables, 43 (2001), 433-449.

[8] C. C. Yang and X. Hua, Unique polynomials of entire and meromorphic functions, Matematicheskaya fizika, analiz, geometriya, 3 (1997), 391-398.

[9] H. Yi, The unique range sets of entire or meromorphic functions, Complex Variables, 28 (1995), 13-21.

[10] H. Yi, Some further results on uniqueness of meromorphic functions, Complex Varialbles, 38 (1999), 375-385.

Department of Mathematics

Faculty of Science

Kanazawa University

Kanazawa, 920-1192

Japan

fujihiro@beach.ocn.ne.jp 